\title{
GRAVITATIONAL SCATTERING EXPERIMENTS
}

\author{
STEPHEN L. W. MCMILLAN \\ Department of Physics, Drexel University, \\ Philadelphía, PA 19104
}

\begin{abstract}
We describe a fully automated gravitational scattering package capable of determining cross sections and reaction rates for binary-singlestar scattering, and present some applications to systems of astrophysical interest.
\end{abstract}

\section{Introduction}

Interactions between binaries and other stars play a central role in determining both the dynamical evolution and the appearance of a globular star cluster. Estimates of the primordial fraction of "hard" binaries (systems with binding energy exceeding the mean stellar kinetic energy, $\frac{3}{2} k T$ ) in globular clusters now range from $\sim 3$ to $\sim 30$ percent, with 10-20 percent regarded as fairly typical (see Hut et al. 1992). The mean time between significant interactions for a $1 k T$ binary is comparable to the local twobody relaxation time, which may be only a few million years in the densest cluster cores. Since mass segregation causes heavier-than-average objects to drift into the high-density central regions on time scales $\sim 1 \mathrm{Gyr}$, we expect binaries in globular clusters to interact frequently with their environment.

Encounters between hard binaries and other cluster members tend to release energy, leaving the binaries more tightly bound and thus heating the cluster (Heggie 1975). Numerical simulations of clusters containing primordial binaries have shown that, so long as any binaries remain in the dynamically "active" energy range (1-50 kT, say, corresponding to orbital semi-major axes of $0.2-10$ A.U. for a cluster 3-D velocity dispersion of 10 $\mathrm{km} \mathrm{s}^{-1}$ ), binaries control the cluster dynamics, and continue to do so until they are all destroyed by interactions with other binaries, or recoil out of the cluster after a triple or four-body encounter (McMillan et al. 1990, 
1991; Gao et al. 1991). For many clusters, this binary depletion time scale may well exceed the age of universe (McMillan \& Hut 1994). In addition to their dynamical importance, binary interactions also greatly increase the probability of stellar collisions, particularly in low-density systems (Hut \& Inagaki 1985; Sigurdsson \& Phinney 1993). A population of $10 \%$ binaries with separations $<1$ A.U. can increase the stellar collision rate by up to two orders of magnitude (Verbunt \& Hut 1987).

While large-scale N-body or Monte-Carlo simulations are probably necessary to study in detail the interplay between the many different physical processes occurring in star clusters, the very comprehensiveness of these approaches makes it difficult to disentangle competing physical effects. Scattering experiments provide an alternative, and much more controlled, means of investigating binary interactions, allowing the investigator to isolate and study specific processes in a systematic manner. The most common way of reporting the outcome of scattering experiments is to quote cross sections for processes of interest. These can then be translated into reaction rates for use in a variety of applications, ranging from heating and interaction rates for Fokker-Planck and Monte-Carlo simulations, to estimates of collision rates and branching probabilities for the production of specific classes of object. The first systematic direct determination of cross sections and reaction rates for binary-single-star encounters was made by Hut \& Bahcall (1983). Recent surveys have been carried out by Hills (1992), Sigurdsson \& Phinney (1993), and Davies (1995).

In the simple case of point-mass (and often identical) stars, it is possible to publish "atlases" of encounter outcomes covering most of the parameter space of interest (see, for example, Hut 1984). However, where there is a distribution of stellar masses, or when stellar evolution and physical interactions between stars must be taken into account, there are simply too many parameter combinations for such atlases to be feasible. Instead, specific questions generally require individual calculations. Rather than having a standard format for reporting cross sections, it is much more useful to have a standard, reliable means of obtaining new results as needed. We describe here a software system for performing these calculations in a robust and efficient manner, automating the many error-prone steps involved in performing a series of scattering calculations.

\section{Computation of Cross Sections}

A scattering experiment entails integrating a large number of individual three-body encounters, holding some parameters fixed and choosing others randomly from specified distributions. In a typical case, the binary semimajor axis $a$ and component masses $m_{1}$ and $m_{2}$ are held fixed, the initial 
mean anomaly is chosen uniformly on $[0,2 \pi)$, while the eccentricity $e$ is either held fixed or chosen randomly from a thermal distribution: $f(e)=2 e$. For the outer orbit, the incomer mass $m_{3}$ and relative velocity at infinity $v_{\infty}$ are specified, the orbital orientation is chosen randomly, and the impact parameter $\rho$ is chosen uniformly in $\rho^{2}$ between $\rho=0$ and $\rho=\rho_{\max }$ (a parameter whose value will be discussed in more detail in a moment). Finally, the initial separation $R_{3}$ is chosen to keep the initial tidal perturbation below some tolerance $\gamma \ll 1: R_{3}=a \gamma^{-1 / 3}\left[1+m_{3} /\left(m_{1}+m_{2}\right)\right]^{1 / 3}$.

If $v_{\infty}$ is less than the critical velocity $v_{c}$, defined by

$$
v_{c}^{2}\left(m_{1}, m_{2}, m_{3}, a\right) \equiv \frac{G m_{1} m_{2}\left(m_{1}+m_{2}+m_{3}\right)}{\left(m_{1}+m_{2}\right) m_{3} a},
$$

then the total energy is negative and the scattering will eventually result in a binary and an unbound single star. In that case, the calculation is terminated when the perturbation on the final binary again falls below $\gamma$. If $v_{\infty} \geq v_{c}$, disruption of the initial binary into three unbound stars is also energetically possible. In that case, the calculation stops when all three stars are receding from one another, are mutually unbound, and none is a significant perturber of the relative motion of the other two.

Encounters are classified as resonances or non-resonances, depending on whether or not the quantity $\left|\mathbf{x}_{1}-\mathbf{x}_{2}\right|^{2}+\left|\mathbf{x}_{2}-\mathbf{x}_{3}\right|^{2}+\left|\mathbf{x}_{3}-\mathbf{x}_{1}\right|^{2}$ has more than one minimum (see Hut 1993). The final state of the system is classified as a preservation, if the initial incomer escapes, an exchange, if one of the binary components escapes, or an ionization, if the binary is destroyed. When nonzero stellar radii are included, physical collisions between stars become possible, and additional final states must be defined.

For uniform sampling with $N$ trials between impact parameters $\rho=0$ and $\rho=\rho_{\max }$, the cross section for events of type $\mathrm{X}$ is simply

$$
\sigma_{X}=\pi \rho_{\max }^{2} \frac{N_{X}}{N}
$$

where $N_{X}$ is the number of times outcome $\mathrm{X}$ occurs. Differential cross sections are determined in an analogous way. The standard error in $\sigma_{X}$ is

$$
\delta \sigma_{X}=\frac{\sigma_{X}}{\max \left(1, \sqrt{N_{X}}\right)} .
$$

In practice, we have found it advantageous to incorporate the determination of $\rho_{\max }$ into the actual calculation of cross sections (rather than relying on inspection of pilot calculations), as follows. We start by performing $n$ scatterings (where the "trial density" $n$ is a parameter controlling the overall accuracy of the final results), uniformly distributed in impact parameter over the range $0 \leq \rho<\rho_{0}=2 a\left[1+G\left(m_{1}+m_{2}+m_{3}\right) / a v_{\infty}^{2}\right]^{1 / 2}$. The 
value of $\rho_{0}$ simply corresponds to a periastron separation of $2 a$. We then systematically expand the impact-parameter range, covering successive annuli of outer radii $\rho_{i}=2^{i / 2} \rho_{0}$ with $n$ trials each, until no "interesting" interactions take place in the outermost zone $\left(i=i_{\max }\right)$ sampled. Typically, an interesting interaction is one in which the binary is significantly perturbed in energy or eccentricity, although the precise definition is easily tailored to the particular application at hand.

This procedure produces rapid convergence toward accurate cross sections, with a minimum of wasted effort. With this non-uniform sampling, expressions (2) and (3) become:

$$
\begin{aligned}
\sigma_{X} & =\frac{\pi}{n} \sum_{i=0}^{i_{\max }}\left(\rho_{i}^{2}-\rho_{i-1}^{2}\right) N_{X i} \\
\left(\delta \sigma_{X}\right)^{2} & =\frac{\pi^{2}}{n^{2}} \sum_{i=0}^{i_{\max }}\left(\rho_{i}^{2}-\rho_{i-1}^{2}\right)^{2} N_{X i}
\end{aligned}
$$

where $N_{X i}$ is the number of times outcome X occurs in zone $i$, and $\rho_{-1}=0$.

\section{The STARLAB Software Environment}

The scattering programs described here operate within a programming environment known as STARLAB, a collection of modular software tools for simulating the evolution of dense stellar systems - star clusters and galactic nuclei-and analyzing the resultant data. It consists of a library of loosely coupled programs, sharing a common data structure, which can be combined in arbitrarily complex ways. Individual modules may be linked in the "traditional" way, as function calls to $\mathrm{C}++$ (the language in which most of the package is written), C, or FORTRAN routines, or at a much higher level-as individual programs connected by UNIX pipes. The former linkage is more efficient, and allows finer control of the package's capabilities; however, the latter provides a quick and compact way of running test simulations. The combination affords great flexibility to STARLAB, allowing it to be used by both the novice and the expert programmer with equal ease. Its structure is described in more detail by McMillan (1996) and McMillan \& Hut (1996).

The automated scattering software in STARLAB is constructed in several layers, each largely independent of the others. The system is designed to function with only high-level user input, but each layer remains accessible if necessary, and diagnostic data at each level can be obtained as desired, without the need for rewriting and recompiling existing sections of code.

The lowest level of the scattering subsystem consists of an orbit integration engine based on a fourth-order, variable-time-step Hermite integrator 
(Makino 1992). The STARLAB scattering integrators augment this algorithm with a novel technique developed by Hut et al. (1995), which guarantees time symmetry in the integration and hence enforces exact energy and momentum conservation in periodic orbits. This results in spectacular improvements in the long-term stability of the integration scheme in all circumstances, even in the case of long-lived resonances.

The next software level consists of several scattering-specific layers. These include: (1) routines to create an initial scattering state, holding some parameters fixed while choosing others randomly, as discussed in $\S 2$; (2) checks to determine whether a given scattering experiment has reached its final outgoing state; (3) optimization features, such as analytical integration of inner and outer orbits of hierarchical triple systems in which the outer orbital period greatly exceeds the inner orbital period, and unperturbed two-body motion near the pericenter of a close encounter; (4) diagnostic functions to store information describing the build-up of energy errors; (5) bookkeeping functions to chart the overall character of the orbits (e.g. "democratic" versus "hierarchical" resonances); (6) checks for stellar collisions, and routines to implement mergers as needed.

Above these layers lies the three-body scattering manager, which oversees the initialization, integration, and classification of a single three-body interaction. The masses and radii of the stars can be specified, as can the orbital parameters of the binary, the impact parameter of the encounter, and the incomer's relative velocity at infinity. The initial distance from which the integration starts is determined automatically, with a default $\gamma$ of $10^{-6}$. Our production runs usually achieve median relative energy errors on the order of $10^{-6}$; however, these could easily be kept as small as $10^{-10}$ if desired.

The next layer contains all the management software needed to conduct a series of scatterings, including the automatic feedback system described earlier to ensure a near-optimal choice of maximum impact parameter. The basic output at this level is a table of total cross sections and errors for all possible outcomes. However, the structure of the package is such that a more ambitious user can use the software as a reliable means of providing a correctly sampled environment in which more complex calculations may be performed, with proper statistical weights attached to each scattering event.

The present highest-level layer is a Maxwellian rate estimator, which uses repeated cross-section calculations to perform a Maxwellian average over velocity. With the complexity of orbit integration and scattering management hidden in the lower-level modules, it is relatively simple to implement new levels, and additional layers can be added with little additional investment in time. 


\section{Sample Applications of the Scattering Software}

\subsection{DETERMINATION OF COLLISION RATES}

The most basic application of the STARLAB scattering package is the generation of total cross sections for specific processes of astrophysical interest. Here we present cross sections for physical collisions and non-colliding exchanges and resonances during encounters between main-sequence binaries and incoming stars, for parameters typical of a globular cluster core.

The binary components are taken to have masses $m_{1}=0.8 M_{\odot}$ and $m_{2}=0.4 M_{\odot}$, and radii $R_{1}=0.8 R_{\odot}$ and $R_{2}=0.4 R_{\odot}$, respectively. The initial binary orbital eccentricity is randomly chosen from a thermal distribution, with the proviso that the separation at periastron is at least twice the sum of the stellar radii, so that immediate collisions are avoided. The incomer is taken to be an intermediate-mass main-sequence star, of mass $m_{3}=0.6 M_{\odot}$, radius $R_{3}=0.6 R_{\odot}$, and velocity at infinity $10 \mathrm{~km} \mathrm{~s}^{-1}$. The results reported here are intended mainly to illustrate the capabilities of the software; they may be compared with similar calculations presented elsewhere in the literature, most recently by Davies (1995).

The outcome of a collision between two main-sequence stars is fairly well known: if the two stars approach within roughly the sum of their radii, they merge to form a single object of approximately double the original radius, with negligible mass loss (see, e.g., Benz \& Hills 1987; Lombardi et al. 1995). In order to determine whether or not a second merger occurs (if the first occurs with the third "spectator" star bound to the center of mass of the colliding pair), we assume the simple mass-radius relation $R \propto m$.

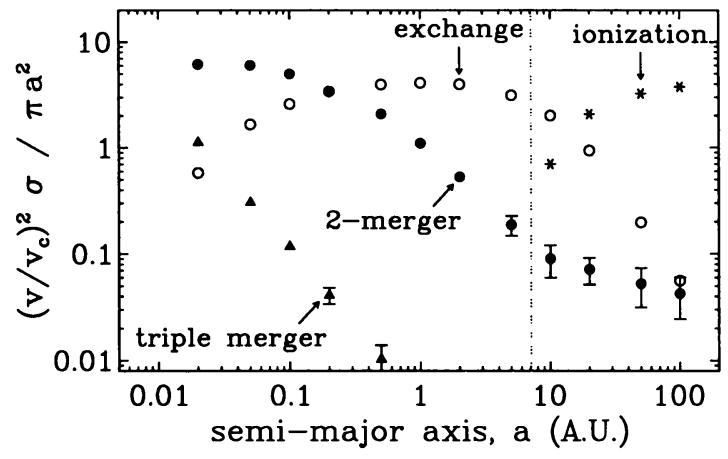

Fig. 1: Scaled cross sections for "clean" exchange (open circles), ionization (stars), two- and threestar mergers (filled circles, filled triangles), for the particular interaction described in the text.

The possible results of an encounter then are: (1) a non-colliding (or "clean") exchange, preservation, or ionization; (2) a two-body merger, leaving a $1.0,1.2$, or $1.4 M_{\odot}$ blue straggler, which may itself be part of a stable binary system; (3) a triple merger, forming a $1.8 M_{\odot}$ blue straggler. Figure 1 shows the cross sections for these processes (excluding preservations, whose cross section is obviously infinite), for binary semi-major axes rang- 
ing from 0.02 to $100 \mathrm{~A}$.U. Ionization occurs only for semi-major axes such that $v_{c}\left(0.8 M_{\odot}, 0.4 M_{\odot}, 0.6 M_{\odot}, a\right)<10 \mathrm{~km} \mathrm{~s}^{-1}$, or $a>7.1 \mathrm{~A} . \mathrm{U}$., as indicated by the dashed line. In this figure, each set of normalized cross sections, along with error estimates, is generated by a single invocation of the cross section calculator described earlier. Here and in Figure 2 below, error bars are shown only where they exceed the size of the symbols used.

The suppression of clean exchanges (whose scaled cross section should be roughly constant in the point-mass limit) by collisions during close encounters is clearly evident in the figure. For the adopted set of initial parameters, collisions dominate over "clean" encounters for $a \lesssim 0.2 \mathrm{~A}$.U., corresponding to initial binary periods of $\lesssim 30$ days. When the factor of $a$ implicit in the $a^{2} v_{c}^{2}$ scaling is taken into account, the 2-body collision cross section is found to be roughly independent of $a$, at $\sim 20$ A.U. ${ }^{2}$ for 0.2 A.U. $\lesssim a \lesssim 10 \mathrm{~A} . \mathrm{U}$. Within this range, the merger ("blue straggler formation") rate per binary in a cluster core of density $10^{4} n_{4}$ stars $\mathrm{pc}^{-3}$ is $\sim 0.05 n_{4} \mathrm{Gyr}^{-1}$. The constancy of the 2-body merger cross section is easily understood as the combination of two factors: the overall binary interaction cross section scales as $a$ because of gravitational focusing, while the probability of a collision during the course of an interaction scales roughly as $\left\langle R_{*}\right\rangle / a$.

The break in the slope of the "2-merger" cross section at $a \sim 10 \mathrm{~A} . \mathrm{U}$. is the result of our particular choice of initial binary eccentricities, which always permitted "almost colliding" systems, regardless of $a$. Collisions with $a \gtrsim 10$ A.U. are mainly "induced mergers," in which the components of a very eccentric binary are perturbed onto a collision course by the passage of the third star. In part because of these induced mergers, collisions between the original binary components tend to dominate; other merger events occur at significant rates only in democratic resonances, and for small $a$.

Figure 2 shows the fraction of two-body mergers resulting in an unbound final system (an isolated blue straggler). Only induced mergers are likely to be unbound; resonant mergers almost always lead to a bound final system. Triple collisions account for only a negligible fraction $(\lesssim 5 \%)$ of the total, except for $a=0.02$ A.U., where they represent about $15 \%$ of all mergers.

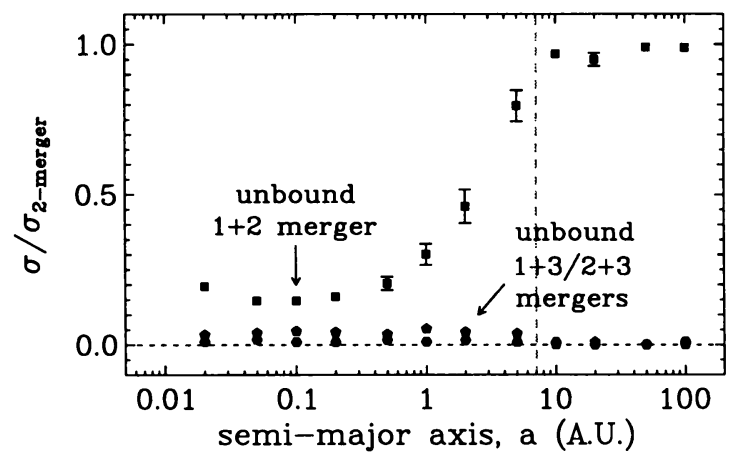

Fig. 2: Branching ratios within the class of twobody mergers (see Fig. 1) for the formation of unbound merger products. Only " $1+2$ " mergers contribute significantly to the total. 


\subsection{FORMATION OF THE TRIPLE SYSTEM B1620-26}

A rather different application of the STARLAB scattering package is described by Rasio, McMillan, \& Hut (1995), who have used the Maxwellian rate estimator to study the "inverse scattering problem" of determining what initial conditions, if any, could have led to the formation of the binary millisecond pulsar (BMP) system B1620-26 in the globular cluster M4. B1620-26 has a low-mass companion (most likely a white dwarf, of mass $m_{2} \approx 0.3 M_{\odot}$ for a pulsar mass $m_{1}=1.35 M_{\odot}$ ) in a nearly circular orbit of period $P_{1}=0.524 \mathrm{yr}$ (Lyne et al. 1988; McKenna \& Lyne 1988), corresponding to a separation $a_{1} \approx 0.8 \mathrm{AU}$. The pulsar timing data indicate the presence of a second, more distant orbital companion (see Backer et al. 1993; Thorsett et al. 1993), with mass in the range $m_{3} \sim 10^{-3}-1 M_{\odot}$, with a corresponding semi-major axis $a_{2} \sim 10-100 \mathrm{AU}$ for the outer orbit (Michel 1994).

We propose that the present triple system could have formed by exchange of an existing BMP into a wide primordial binary. The motivation for invoking such an explanation is as follows. If the second pulsar companion is indeed a main-sequence star of mass $\sim 0.5 M_{\odot}$, then the eccentricity of the outer orbit must be large $\left(e_{2}>0.5\right)$ and its semi-major axis cannot be much smaller than 50-100 A.U., to be consistent with the pulsar timing data (Rasio 1994). Such a wide orbit is easily disrupted at its location near the core of M4: we estimate its lifetime to be $\sim 10^{7}-10^{8} \mathrm{yr}$, much shorter than the age of the BMP, which is most likely $\gtrsim 10^{9} \mathrm{yr}$ (see Thorsett et al. 1993). Thus the pulsar could not have formed inside the triple system. Instead, the BMP must have become a member of the triple by exchange after its formation.

This scenario is supported by the tentative identification by Bailyn et al. (1994) of an optical counterpart to the triple companion, with mass $\sim 0.5 M_{\odot}$. For the most likely parameters of the outer orbit, the perturbation on the inner binary due to the triple distant companion can also account for the binary's unexpectedly large eccentricity of 0.025 .

We thus wish to determine which primordial binary parameters could have led to an exchange interaction that neither disrupted the wide primordial binary, nor perturbed the BMP to an eccentricity greater than actually observed. Since the eccentricity perturbation decreases sharply with increasing separation, this in effect means that no star approached within $\sim 3 a_{1}$ of the BMP during the encounter. In the terminology of the previous section, we seek a clean exchange, with the BMP treated as a single particle of radius $3 a_{1}$. We find that such an exchange is in fact the most likely outcome of the interaction for primordial binary semi-major axes $a_{M S} \sim 10-30$ A.U. and binary component masses $\sim 0.3-0.8 M_{\odot}$. 
Figure 3 shows the joint distribution of $a_{2}$ and $e_{2}$ for one particular choice of primordial binary parameters $\left(a_{M S}=13\right.$ A.U., each component mass $0.5 M_{\odot}$ ). Clearly, the proposed mechanism is capable of forming triples with the desired characteristics: a nearly circular inner orbit (that remains unperturbed after the interaction) and a very eccentric outer orbit with $a_{2} \sim 10^{2}$ A.U. The derived rate for the formation of a triple similar to B1620-26 is also consistent with the observation of one such object in the entire Galactic globular cluster system.

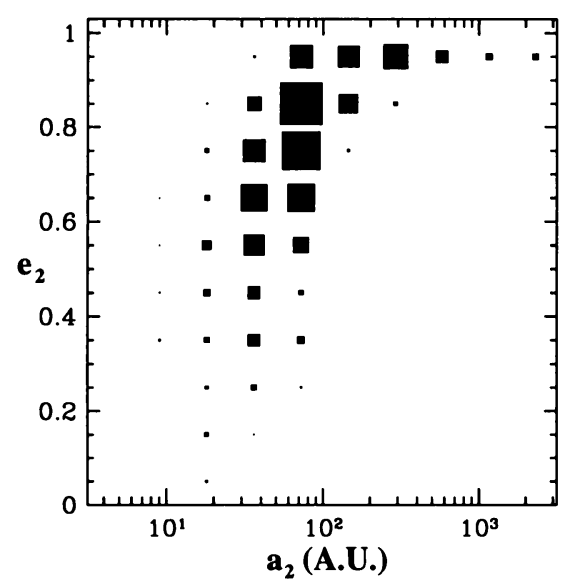

Fig. 3: Distribution of the semi-major axis $a_{2}$ and eccentricity $e_{2}$ of the triple's outer orbit. The size of each square dot is proportional to the differential cross-section for producing a triple system with those specific orbital parameters.

\subsection{EXCHANGE CROSS SECTIONS FOR ARBITRARY MASSES}

Finally, Heggie, Hut, \& McMillan (1996) have used the STARLAB software in fully automated mode as the basis for an extensive calculation of exchange cross sections. In the limit of very hard binaries, $v_{\infty} \ll v_{c}$, the overall scaling of the exchange cross section can be shown to be

$$
\sigma \sim \sigma_{0}=\frac{G M_{123} a}{v_{\infty}^{2}}\left(\frac{M_{23}}{M_{123}}\right)^{1 / 6}\left(\frac{m_{3}}{M_{13}}\right)^{7 / 2}\left(\frac{M_{123}}{M_{12}}\right)^{1 / 3}\left(\frac{M_{13}}{M_{123}}\right)
$$

where $M_{12}=m_{1}+m_{2}$, etc., $M_{123}=m_{1}+m_{2}+m_{3}$, and we have adopted the convention that binary component 1 is ejected following the encounter. The above expression is asymptotically valid in all regimes where one star's mass dominates the total, or is negligible.

By using STARLAB to determine the exchange cross sections on a grid of points in $\left(0 \leq \mu_{1} \equiv m_{1} / M_{12} \leq 1,0 \leq \mu_{2} \equiv m_{3} / M_{123} \leq 1\right)$, then fitting the results to an expression of the form $\sigma=\sigma_{0} f\left(\mu_{1}, \mu_{2}\right)$, we have obtained a fitting formula valid for arbitrary masses, and accurate to better than $\sim 20 \%$ over most of parameter space of interest. For more details, and for the final fitting formula, see Heggie et al. (1996). 


\section{Outlook}

The design of STARLAB facilitates inclusion of more detailed physical processes into our models, and this represents one obvious direction of future development of the system. In addition, binary-binary and general $N$-body scattering packages are nearing completion. While more complex, their conceptual framework is similar to that described here for the 3 -body case. The entire package is available by anonymous FTP from ftp.sns.ias.edu/pub/starlab, or via http://www.sns.ias.edu/ starlab. STARLAB has been successfully installed on UNIX systems running SunOS 4, Solaris 2, HP-UX, Linux, and Dec OSF, using both native C++ compilers and the GNU g++ compiler. Real-time demonstrations of the software are available through the World-Wide Web site listed above.

This work was supported in part by NASA grant NAGW-2559 and NSF grant AST-9308005.

\section{References}

Backer, D.C., Foster, R.S., \& Sallmen, S. 1993, Nature, 365, 81

Bailyn, C.D., Rubenstein, E.P., Girard, T.M., Dinescu, D., Rasio, F.A., \& Yanny, B. 1994, ApJL, 433, L89

Benz, W., \& Hills, J.G. 1987, ApJ, 323, 614

Davies, M.B. 1995, MNRAS, 276, 887

Gao, B., Goodman, J.G., Cohn, H.N., \& Murphy, B. 1991, ApJ, 370, 567

Heggie, D.C. 1975, MNRAS, 173, 729

Heggie, D.C., McMillan, S.L.W., \& Hut, P. 1996, these proceedings; submitted to ApJ

Hills, J.G. 1992, AJ, 103, 1955

Hut, P. 1984, ApJS, 55, 301

Hut, P. 1993, ApJ, 403, 256

Hut, P. \& Bahcall, J. N. 1983, ApJ, 268, 319

Hut, P., \& Inagaki, S. 1985, ApJ, 298, 502

Hut, P., McMillan, S.L.W., Goodman, J.G., Mateo, M., Phinney, E.S., Pryor, C., Richer, H.B., Verbunt, F., \& Weinberg, M. 1992, PASP, 105, 981

Hut, P., Makino, J., \& McMillan, S.L.W. 1995, ApJL, 443, L93

Lombardi, J.C., Rasio, F.A., \& Shapiro, S.L. 1995, preprint

Lyne, A., Biggs, J., Brinklow, A., Ashworth, M., \& McKenna, J. 1988, Nature, 332, 45

Makino, J. 1992, ApJ, 369, 200

McMillan, S.L.W. 1996, in Binaries in Star Clusters, ed. G. Milone (San Francisco: ASP)

McMillan, S.L.W., Hut, P., \& Makino, J. 1990, ApJ, 362, 522

McMillan, S.L.W., Hut, P., \& Makino, J. 1991, ApJ, 372, 111

McMillan, S.L.W., \& Hut, P. 1994, ApJ, 427, 793

McMillan, S.L.W., \& Hut, P. 1996, submitted to ApJ

McKenna, J., \& Lyne, A.G. 1988, Nature, 336, 226; erratum, 336, 698

Michel, F.C. 1994, in Millisecond Pulsars: The Decade of Surprise, ed. Fruchter et al. (San Francisco: ASP)

Rasio, F.A. 1994, ApJL, 427, L107

Rasio, F.A., McMillan, S.L.W., \& Hut, P. 1995, ApJL, 438, L33

Sigurdsson, S., \& Phinney, E.S. 1993, ApJ, 415, 631

Thorsett, S.E., Arzoumanian, Z., \& Taylor, J.H. 1993, ApJL, 412, L33

Verbunt, F., \& Hut, P. 1987, in The Origin and Evolution of Neutron Stars, eds. D. J. Helfand, and J. H. Huang (Dordrecht: Reidel), p. 187 